\title{
Topology and Wilson lines: global aspects of the double copy
}

\author{
Luigi Alfonsi, Chris D. White and Sam Wikeley \\ Centre for Research in String Theory, School of Physics and Astronomy, \\ Queen Mary University of London, 327 Mile End Road, London E1 4NS, U.K. \\ E-mail: 1.alfonsi@qmul.ac.uk, christopher.white@qmul.ac.uk, \\ s.wikeley@qmul.ac.uk
}

ABSTRACT: The Kerr-Schild double copy relates exact solutions of gauge and gravity theories. In all previous examples, the gravity solution is associated with an abelian-like gauge theory object, which linearises the Yang-Mills equations. This appears to be at odds with the double copy for scattering amplitudes, in which the non-abelian nature of the gauge theory plays a crucial role. Furthermore, it is not yet clear whether or not global properties of classical fields - such as non-trivial topology — can be matched between gauge and gravity theories. In this paper, we clarify these issues by explicitly demonstrating how magnetic monopoles associated with arbitrary gauge groups can be double copied to the same solution (the pure NUT metric) in gravity. We further describe how to match up topological information on both sides of the double copy correspondence, independently of the nature of the gauge group. This information is neatly expressed in terms of Wilson line operators, and we argue through specific examples that they provide a useful bridge between the classical double copy and the BCJ double copy for scattering amplitudes.

Keywords: Scattering Amplitudes, Wilson, 't Hooft and Polyakov loops

ArXiv EPrint: 2004.07181 


\section{Contents}

1 Introduction $\quad 1$

2 The Kerr-Schild double copy 3

3 (Non)-abelian magnetic monopoles and their topology 5

3.1 Abelian case 5

3.2 Non-abelian case 8

3.2.1 Gauge field of a non-abelian monopole $\quad 8$

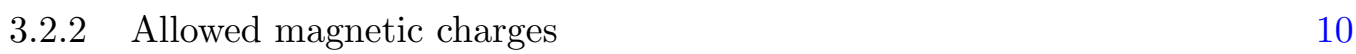

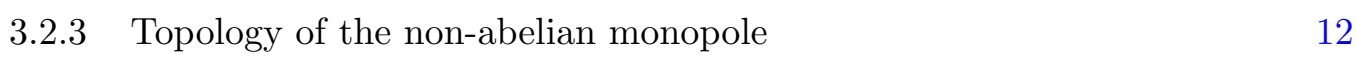

4 Topology of the Taub-NUT solution $\quad 13$

5 Wilson lines and the double copy $\quad 18$

5.1 The all-order structure of infrared singularities 20

5.2 The Regge limit 21

6 Conclusion $\quad 22$

A Topological classification of $\mathrm{SU}(N) / \mathbb{Z}_{N}$ bundles 23

\section{Introduction}

Since its inception just over a decade ago [1-3], the double copy has remained an intensivelystudied relationship between gauge and gravity theories. Its first application was to scattering amplitudes, and there is by now a large amount of evidence for its validity at arbitrary loop orders [2, 4-43], either with or without supersymmetry. The double copy has also been extended to classical solutions [44-81], some of which are exact solutions of their respective field equations. There are ongoing hopes that this may provide new calculational tools for classical General Relativity, including for astrophysical applications such as gravitational wave detection. In parallel to this effort, however, it is worth examining the conceptual underpinnings of the double copy correspondence, and to try to ascertain the limits (if any) of its validity. If, for example, the double copy can be extended to arbitrary (non)perturbative solutions in gauge and gravity theories, this clearly indicates a profound new correspondence between different quantum field theories, that may even suggest that our traditional approach to thinking about field theory needs revisiting. One way to probe this is to find explicit non-perturbative solutions in theories that enter the double copy correspondence, such as the biadjoint scalar theory considered in refs. [82-85]. 


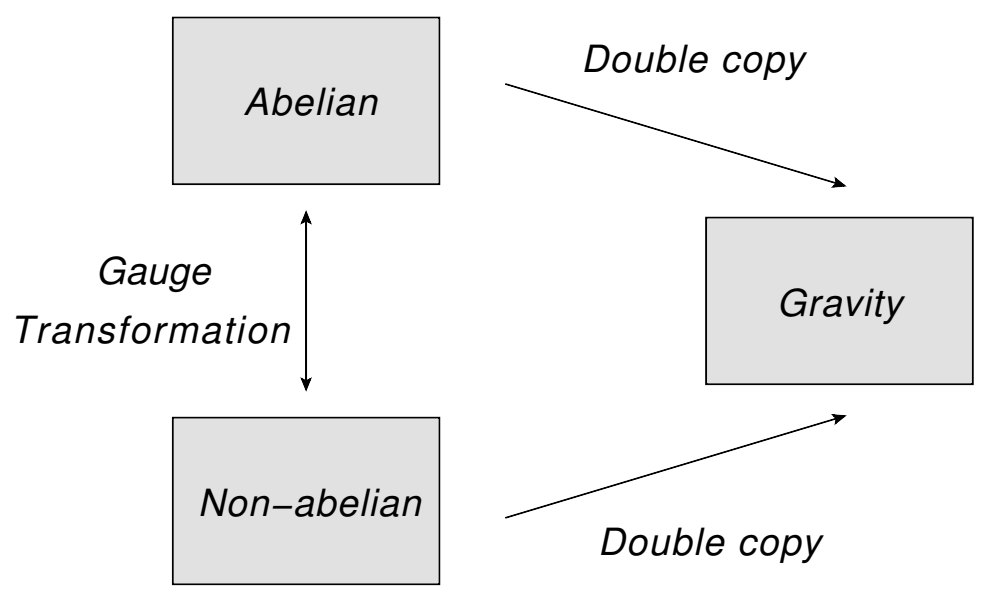

Figure 1. Conceptual structure of the double copy for exact solutions, in which (non-)abelian objects must double copy to the same gravity solution, regardless of the gauge group.

There is, however, another approach to extending the conceptual framework of the double copy. This is to note that nearly all previous incarnations in either a classical or quantum context have involved local quantities. Scattering amplitudes, for example, have locality built in, even if this is not manifest. Furthermore, the classical double copy initiated in ref. [44] involves products of fields at the same spacetime point, and thus is again manifestly local. If it is true that there is a deep connection between gauge and gravity theories, it must be possible to match up global data - such as topological information - between them. This was examined recently in ref. [86] which considered the single copy of the Eguchi-Hanson instanton, alas without providing any conclusive evidence that topological information can be double-copied. Nevertheless, the idea clearly deserves further investigation, and is thus the main aim of this paper.

For scattering amplitudes and (non-linear) perturbative classical solutions, the nonabelian nature of the gauge theory plays a crucial role in the formulation of the double copy procedure. As is by now well-known, there exists a special class of gravity solutions - time-independent Kerr-Schild metrics - whose single copy gauge solution obeys the linearised Yang-Mills equations, and thus looks like an abelian gauge theory solution [44]. In what sense the gauge theory solution is genuinely non-abelian is then rather obscure. However, by analogy with amplitudes we must be able to find a construction such that exact classical solutions with different gauge groups map to the same gravity solution, as shown in figure 1. This follows from the fact that colour information is removed when performing the double copy, so that a given gravity solution does not care which gauge group one started with. Indeed, figure 1 has a precedent in the study of infrared singularities of amplitudes [27], and was also considered for solutions of $\mathrm{SU}(2)$ gauge theory in ref. [85] (see refs. [35, 36] for yet more examples). However, this would appear to forbid the ability to map topological information between gauge and gravity solutions: topological invariants (e.g. characteristic classes) of a gauge field usually rely crucially on which particular gauge group one considers, and this information is irrelevant after taking the double copy.

In this paper, we will see that it is indeed possible to relate exact solutions with nontrivial topology between gauge and gravity theories in a meaningful way. First, we will 
take a particular solution of pure gauge theory, namely a (singular) point-like magnetic monopole. For general gauge groups, this is known to have a non-trivial topology, where the latter may or may not be classified by a known topological invariant. However, it can always be characterised by a certain patching condition between gauge fields in different spatial regions. Furthermore, the non-abelian monopole can always be written in a gauge in which it takes the form of a trivially dressed abelian-like monopole solution, whose double copy is already known [45] to be the Taub-NUT solution [87, 88]. There is an established patching condition for the Taub-NUT metric that characterises its non-trivial topology [89], and we will see that this precisely corresponds to the similar condition in gauge theory, independently of the nature of the gauge group. Thus, we provide an explicit example of an exact solution whose local and global properties obey the construction of figure 1, and which fully generalises the preliminary observations regarding $\mathrm{SU}(2)$ monopoles made recently in ref. [85]. Furthermore, we will see that all of our patching conditions - in either gauge or gravity theories - can be expressed in terms of certain Wilson line operators. This will allow us to make contact between the study of magnetic monopoles in this paper, and previous results concerning the structure of scattering amplitudes in special kinematic limits [27, 29].

The structure of this paper is as follows. In section 2, we briefly review the KerrSchild double copy. In section 3, we examine (non-)abelian monopoles, and describe the characterisation of their non-trivial topology. In section 4, we study the topology of TaubNUT spacetime, and relate this to the single copy gauge theory solutions considered in section 3. The relevance and implications of Wilson lines will be outlined in section 5 . Finally, we discuss our results and conclude in section 6 .

\section{The Kerr-Schild double copy}

The aim of the classical double copy is to associate a given non-abelian gauge theory solution with a gravitational counterpart, in a way that overlaps with the known BCJ double copy for scattering amplitudes where appropriate [1-3]. For general solutions, this must be carried out order-by-order in the relevant coupling constants. However, a certain special family of exact gravity solutions is known, which all have well-defined gauge theory single copies. These are the Kerr-Schild metrics (see e.g. [90] for a modern review), which can be defined as follows: ${ }^{1}$

$$
g_{\mu \nu}=\eta_{\mu \nu}+\kappa h_{\mu \nu}, \quad h_{\mu \nu}=\phi k_{\mu} k_{\nu} .
$$

Here $h_{\mu \nu}$ is the graviton field representing the deviation from the Minkowski metric $\eta_{\mu \nu}$, and $\kappa=\sqrt{32 \pi G_{N}}$, where $G_{N}$ is the Newton constant. The Kerr-Schild form corresponds to the graviton decomposing into a scalar field $\phi$ multiplying an outer product of a vector $k_{\mu}$ with itself, where the latter must satisfy null and geodesic properties:

$$
\eta_{\mu \nu} k^{\mu} k^{\nu}=g_{\mu \nu} k^{\mu} k^{\nu}=0, \quad k \cdot \partial k^{\mu}=0 .
$$

\footnotetext{
${ }^{1}$ Note that we use the $(+,-,-,-)$ metric signature throughout.
} 
This ansatz turns out to linearise the Einstein equations, such that exact solutions can be more easily found. Each such solution is characterised by the explicit forms of $\phi$ and $k_{\mu}$, and ref. [44] proved that for every time-independent Kerr-Schild graviton, one may then construct a single copy gauge field

$$
\mathbf{A}_{\mu}=\left(c^{a} \mathbf{T}^{a}\right) \phi k_{\mu}
$$

which obeys the linearised Yang-Mills equations. Here $c^{a}$ is an arbitrary constant colour vector, and $\mathbf{T}^{a}$ is a generator of the gauge group. The extension to symmetries other than time translational invariance has been considered in ref. [91]. Clearly eq. (2.3) is not unique: for a particular gauge group, any choice of $c^{a}$ will suffice. We may furthermore pick any gauge group. Indeed, it is instructive to write eq. (2.3) as

$$
\mathbf{A}_{\mu}=\left(c^{a} \mathbf{T}^{a}\right) A_{\mu}^{\text {abel. }},
$$

where $A_{\mu}^{\text {abel. }}$ is the solution of a purely abelian gauge theory. If the latter has a known Kerr-Schild double copy, any solution of the form of eq. (2.4), in which the colour structure completely factorises, can also be easily double copied. One simply strips off the colour charge $\left(c^{a} \mathbf{T}^{a}\right)$, and double copies the gauge field as if it were abelian. Note that this provides an explicit realisation of figure 1 , in which both abelian and non-abelian classical solutions are taken to map to the same gravity solution.

There is a growing list of specific cases of the Kerr-Schild double copy which have been examined in detail. Arguably the simplest is that of a point-like mass $M$ in gravity giving rise to the Schwarzschild metric, for which the single copy is a point-like (electric) charge [44]. ${ }^{2}$ Particularly relevant for this paper is the Taub-NUT solution in gravity [87, 88], which was examined from a double copy point of view in ref. [45]. This has a Schwarzschild-like mass term, but in addition a so-called NUT charge $N$, which gives rise to a rotational character in the gravitational field at infinity. In particular (Plebanski) coordinates, the relevant graviton field can be written in a so-called double Kerr-Schild form [92]:

$$
h_{\mu \nu}=M \phi k_{\mu} k_{\nu}+N \psi l_{\mu} l_{\nu}
$$

where $\phi$ and $\psi$ are distinct scalar fields, and $k_{\mu}, l_{\mu}$ different vectors that obey certain mutual orthogonality conditions. Such an ansatz will not linearise the Einstein equations in general, but happens to do so for Taub-NUT. One may then single copy the solution to create a gauge field

$$
\mathbf{A}_{\mu}=\left(c^{a} \mathbf{T}^{a}\right) \phi k_{\mu}+\left(\tilde{c}^{a} \mathbf{T}^{a}\right) \psi l_{\mu} .
$$

Reference [45] analysed this solution in an abelian gauge theory, and concluded that it was a dyon, possessing both electric and magnetic monopole charge. These are represented by the first and second terms in eq. (2.6) respectively, such that for the case of a pure NUT charge in the gravity theory, one may follow eq. (2.4) and write

$$
\mathbf{A}_{\mu}=\left(\tilde{c}^{a} \mathbf{T}^{a}\right) A_{\mu}^{\mathrm{D}}
$$

\footnotetext{
${ }^{2}$ Going the other way, the double copy of a point charge may be more general than a pure gravity solution, as discussed in refs. [57, 60, 78].
} 
where ${ }^{3}$

$$
A_{\mu}^{D}=-\tilde{g}(0,0,0, \cos \theta-1)
$$

is the well-known gauge potential for a Dirac magnetic monopole in the U(1) (abelian) gauge theory of electromagnetism. We have here expressed the result in spherical polar coordinates $(t, r, \theta, \varphi)$, and denoted the magnetic charge parameter that would arise in a purely abelian theory by $\tilde{g}$. Reference [45] did not explicitly check that eq. (2.7) represents a genuine magnetic monopole solution in the case of a non-abelian gauge theory. We will see in the following section that this is indeed the case, such that $\tilde{g}\left(\tilde{c}^{a} \mathbf{T}^{a}\right)$ is the appropriate non-abelian generalisation of the magnetic charge. In line with the above comments, the double copy of eq. (2.7) is straightforward, and always results in a pure NUT charge in gravity.

\section{3 (Non)-abelian magnetic monopoles and their topology}

In the previous section, we have seen that (non)-abelian magnetic monopoles with arbitrary gauge groups all map to a pure NUT charge in gravity. In this section, we discuss how to characterise the non-trivial topology of monopole solutions, in both abelian and non-abelian gauge theories.

\subsection{Abelian case}

The gauge potential for the Dirac monopole of eq. (2.8) has a singularity at $\theta=\pi$, corresponding to the entire negative $z$-axis, which is usually referred to as the Dirac string. Whilst it is not possible to remove the physical singularity at $r=0$ (i.e. the location of the singular point-like monopole), the orientation of the string singularity can be modified by performing a gauge transformation. To construct a gauge field that is everywhere nonsingular away from the origin, one must employ at least two coordinate patches, such that the gauge field is non-singular in each. Where these coordinate patches overlap the gauge fields defined in each can then be glued together.

This idea was first discussed by $\mathrm{Wu}$ and Yang, who gave an elegant fibre bundle interpretation [93]. To briefly summarise for the uninitiated: gauge fields can be defined as connections on principal fibre bundles, namely manifolds that look locally like a product space $\mathcal{M} \times G$, where $\mathcal{M}$ denotes Minkowski spacetime, and $G$ the gauge group. One refers to $\mathcal{M}$ as the base space and $G$ as the fibre, where there is one fibre attached to each point in the base space. Whilst such a bundle may look locally like a product space, it might differ from this globally. Thus, for a given gauge group, there may be more than one fibre bundle with the same local structure. The classification of topologically non-trivial gauge fields is then entirely equivalent to the classification of fibre bundles.

The Dirac monopole is singular at the origin and thus the base space is Minkowski spacetime with the origin removed, $\mathcal{M}-\{0\}$. The spatial part of this manifold can be

\footnotetext{
${ }^{3}$ Here, and for the rest of the paper, spherical coordinates are defined such that

$$
A_{x} d x+A_{y} d y+A_{z} d z=A_{r} d r+A_{\theta} d \theta+A_{\phi} d \phi .
$$
}




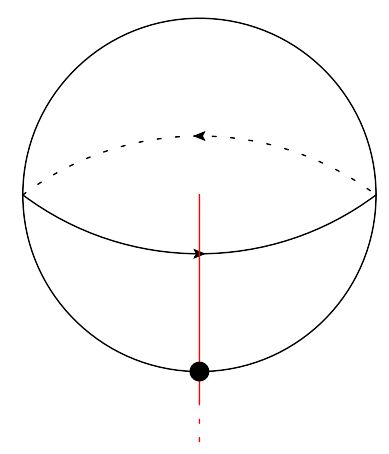

(a)

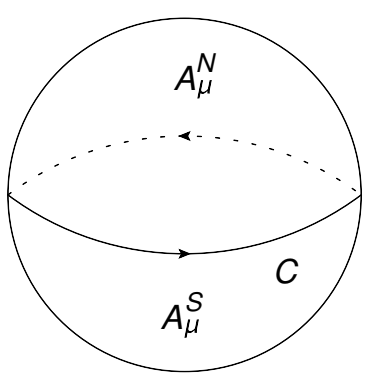

(b)

Figure 2. (a) The Dirac monopole field of eq. (2.8) has a string-like singularity that cuts the sphere at infinity at the south pole; (b) Wu-Yang construction of a non-singular gauge field: one may take non-singular gauge fields in the northern and southern hemispheres, such that they are patched together by a gauge transformation on the equator $C$.

continuously deformed into the surface of a sphere at infinity. Adding the time direction, the base space is now topologically equivalent to $S^{2} \times \mathcal{R}$. The monopole field of eq. (2.8) is tangential to the surface of the sphere, and it is well known that it is impossible to define a vector field on $S^{2}$ without it being ill-defined at one point. This itself explains the presence of the Dirac string, which may be taken to puncture the sphere at infinity at the singular point, as shown in figure 2 .

We indeed then need at least two coordinate patches in order to define the gauge field throughout all space, which we can take to be the northern and southern hemispheres respectively. We are free to define separate gauge fields $A_{\mu}^{N}$ and $A_{\mu}^{S}$ in each patch, as shown in figure 2, provided that they are related by a gauge transformation in the region of overlap, namely on the equator $C$. This is straightforward to achieve in practice. Firstly, the field of eq. (2.8) is already non-singular in the northern hemisphere, and so can be taken as $A_{\mu}^{N}$. One may then define the gauge field

$$
A_{\mu}^{S}=-\tilde{g}(0,0,0, \cos \theta+1) .
$$

This is related to $A_{\mu}^{N}$ by the gauge transformation

$$
A_{\mu}^{S}=A_{\mu}^{N}-\frac{i}{g} S(\varphi) \partial_{\mu} S^{-1}(\varphi)
$$

where $g$ is the coupling constant, and

$$
S(\varphi)=e^{2 i g \tilde{g} \varphi}
$$

is an element of the gauge group. The field of eq. (3.1) has a string-like singularity on the positive $z$ axis, and is thus non-singular throughout the southern hemisphere as required. Furthermore, the two fields can be matched at the equator, where they are equivalent up to the gauge transformation in eq. (3.2). This completes the construction of a single-valued, non-singular gauge field away from the origin. 
The patching together of two distinct gauge fields constitutes a non-trivial topology, which turns out to be related to the magnetic charge. To see this, note that the magnetic flux is given by

$$
\Phi_{B}=\iint_{S} F_{\mu \nu} d \Sigma^{\mu \nu}
$$

where $d \Sigma^{\mu \nu}$ is the area element on the surface $S$ corresponding to the 2 -sphere at infinity. Separating the surface integral into two separate contributions for the northern and southern hemispheres, one may apply Stokes' theorem to rewrite eq. (3.4) as

$$
\Phi_{B}=\oint_{C} d x^{\mu}\left(A_{\mu}^{N}-A_{\mu}^{S}\right)=4 \pi \tilde{g}
$$

where we have used eqs. (3.2), (3.3). This justifies the statement that $\tilde{g}$ represents the amount of magnetic charge. Furthermore, the requirement that the gauge transformation of eq. (3.2) be single-valued (i.e. that $S(2 \pi)=S(0)$ in eq. (3.3)) implies the famous Dirac quantisation condition

$$
g \tilde{g}=\frac{n}{2}, \quad n \in \mathbb{Z},
$$

which relates the electric and magnetic charge. The relation to the topology of the fibre bundle can be seen from the fact that there are discretely different $\mathrm{U}(1)$ principle bundles, each of which is classified by the so-called first Chern number. The latter, up to a constant factor, is given precisely by eq. (3.4).

There is another way to derive the quantisation condition, which makes the relevant topological aspects clearer, and which will also pave the way for the non-abelian discussion in the following section. Consider the family of circles $C(\theta)$ on the 2 -sphere at infinity ${ }^{4}$ characterised by constant values of the polar angle $\theta$. One may associate each such curve with a group element as follows:

$$
U(\theta)=\exp \left[i g \oint_{C(\theta)} d x^{\mu} A_{\mu}\right] .
$$

At $\theta=0$, the curve $C(0)$ is an infinitesimally small loop around the north pole, and thus $U(0)$ corresponds to the identity element of the gauge group. As $\theta$ increases from 0 to $\pi$, the curves gradually sweep over the sphere as shown in figure 3(a), culminating in an infinitesimally small loop at the south pole, such that $U(\pi)$ also corresponds to the identity element. At $\theta=\pi / 2$, the definition of the gauge field jumps from the northern field $A_{\mu}^{N}$ to the southern field $A_{\mu}^{S}$. We may depict this as shown in figure 3(b). The curve obtained in the gauge group as $\theta$ increases from zero proceeds clockwise from the lower point as shown, reaching point $A$ at $\theta=\pi / 2$. Upon switching to the southern gauge field, there is a discontinuity and the curve continues from point $B$. However, eq. (3.7) represents the phase that a charged particle would experience upon traversing the curve $C(\theta)$. For this to be single-valued on the equator, it follows that the points $B$ and $A$ should correspond to equivalent group elements (indicated by the dotted line). Thus, as $\theta$ varies in the range $[0, \pi]$, a closed curve is swept out in the gauge group $G$. The set of topologically non-trivial

\footnotetext{
${ }^{4}$ In fact, any sphere surrounding the origin will do e.g. ref. [93] uses a unit radius.
} 


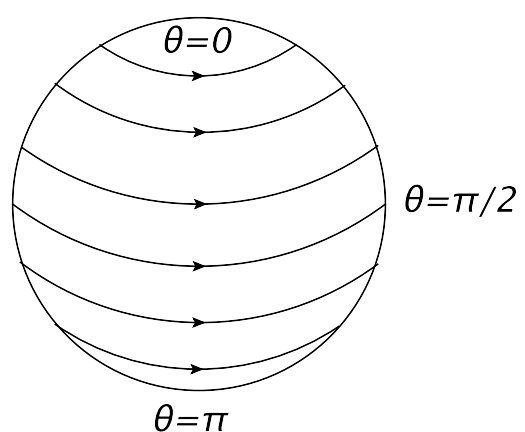

(a)

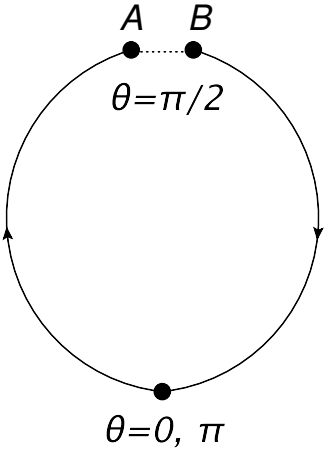

(b)

Figure 3. (a) A family of curves for fixed values of the polar angle $\theta$, where $\theta=0$ and $\theta=\pi$ correspond to the north and south poles respectively; (b) closed loop in the gauge group $G$ associated with the family of curves.

gauge fields is then in one-to-one correspondence with the set of topologically inequivalent closed loops in $G$, namely the possible ways in which one can join the points $A$ and $B$ in figure 3(b). These closed loops are classified by the first homotopy group $\pi_{1}(G)$, where for the present case one has

$$
\pi_{1}(\mathrm{U}(1))=\mathbb{Z}
$$

i.e. the set of integers under addition. This simple result follows from the fact that the group manifold of $\mathrm{U}(1)$ is a circle, such that any closed loop may wind around this circle an integer number of times. Put another way, the fact that the two points $A$ and $B$ in figure $3(\mathrm{~b})$ correspond to equivalent group elements imposes — via eq. (3.7) - the condition

$$
g \oint_{C(\theta)} d x^{\mu} A_{\mu}^{N}=g \oint_{C(\theta)} d x^{\mu} A_{\mu}^{S}+2 \pi n, \quad n \in \mathbb{Z},
$$

which is precisely equivalent to eqs. (3.5), (3.6).

\subsection{Non-abelian case}

In this section, we review the properties of singular point-like non-abelian magnetic monopoles, where we will follow the presentation of ref. [94] closely. Our ultimate aim is to identify the relevant quantity that specifies their non-trivial topology, and to interpret this from the point of view of the double copy. ${ }^{5}$ First, let us address the form of a non-abelian monopole field $\mathbf{A}_{\mu}=A_{\mu}^{a} \mathbf{T}^{a}$.

\subsubsection{Gauge field of a non-abelian monopole}

Assuming a static field, we may choose a gauge such that $A_{0}^{a}=0$. The remaining components of the gauge field can then be expanded in inverse powers of the radial coordinate:

$$
A_{i}^{a}=\frac{a_{i}^{a}(\theta, \phi)}{r}+\mathcal{O}\left(r^{-2}\right),
$$

\footnotetext{
${ }^{5}$ Most of the literature on non-abelian monopoles focuses on spontaneously broken theories with additional scalar fields, due to the non-singular nature of the solutions. For useful reviews of the singular case, see e.g. refs. [94, 95].
} 
where $A_{i}^{a}$ and $a_{i}^{a}$ belong to the Lie algebra of $G$. This gives rise to a magnetic field

$$
B_{i}^{a}=-\frac{1}{2} \epsilon^{i j k} F_{j k}^{a}
$$

where the field strength $\mathbf{F}_{\mu \nu}=F_{\mu \nu}^{a} \mathbf{T}^{a}$ is defined by

$$
\mathbf{F}_{\mu \nu}=\partial_{\mu} \mathbf{A}_{\nu}-\partial_{\nu} \mathbf{A}_{\mu}-i g\left[\mathbf{A}_{\mu}, \mathbf{A}_{\nu}\right] .
$$

For a point-like monopole solution we require a magnetic field with $r^{-2}$ dependence, and therefore the $\mathcal{O}\left(r^{-2}\right)$ terms in the potential can be omitted. To avoid any issues arising from the unavoidable singularity at the origin, we consider the field only for $r>r_{0}$, where $r_{0}$ is the small but non-vanishing radius of a sphere centred on the monopole. To simplify the analysis, we first choose a gauge in which $A_{r}=0$. This can be done by finding a gauge transformation such that

$$
\mathbf{A}_{r} \rightarrow \mathbf{U} \mathbf{A}_{r} \mathbf{U}^{-1}+\mathbf{U} \partial_{r} \mathbf{U}^{-1}=0
$$

A solution to this expression is

$$
\mathbf{U}^{-1}(r, \theta, \phi)=\mathcal{P} \exp \left[\frac{i}{g} \int_{r_{0}}^{r} \mathrm{~d} r^{\prime} \mathbf{A}_{r}\left(r^{\prime}, \theta, \phi\right)\right],
$$

where $\mathcal{P}$ denotes path ordering. This describes an integration along radial lines, with the lower bound protecting the integral from the singularity at the origin. A similar procedure can be implemented for $\mathbf{A}_{\theta}$ by integrating along lines of constant $r$ and $\phi$, resulting in a gauge in which $\mathbf{A}_{\theta}=0$. This leaves $\mathbf{A}_{\phi}$ as the only non-zero component of the gauge field, the form of which may be determined from the equations of motion. The monopoles considered here are time-independent, so the $\mathbf{F}_{0 i}$ terms in the field strength of eq. (3.12) vanish. Furthermore, the assumptions made thus far imply that at large distances $\mathbf{A}_{\phi}$ is independent of $r$. Hence, the only non-vanishing component of the field strength is

$$
\mathbf{F}_{\theta \phi}=\partial_{\theta} \mathbf{A}_{\phi}
$$

The Yang-Mills field equations in spherical coordinates are

$$
\partial_{\mu} \sqrt{\eta} \mathbf{F}^{\mu \nu}-i g\left[\mathbf{A}_{\mu}, \sqrt{\eta} \mathbf{F}^{\mu \nu}\right]=0,
$$

where $\eta$ is the absolute value of the determinant of the Minkowski metric. The field equations give rise to two non-trivial equations of motion:

$$
\begin{aligned}
\partial_{\theta} \sqrt{\eta} \mathbf{F}^{\theta \phi} & =0, \\
\partial_{\phi} \sqrt{\eta} \mathbf{F}^{\phi \theta}-i g\left[\mathbf{A}_{\phi}, \sqrt{\eta} \mathbf{F}^{\phi \theta}\right] & =0 .
\end{aligned}
$$

Equation (3.17) results in

$$
\partial_{\theta}\left(\frac{1}{\sin \theta} \partial_{\theta} \mathbf{A}_{\phi}\right)=0
$$


which admits a general solution

$$
\mathbf{A}_{\phi}=\mathbf{M}(\phi)+\frac{\mathbf{Q}_{M}(\phi)}{4 \pi} \cos \theta
$$

where $\mathbf{M}(\phi)$ and $\mathbf{Q}_{M}(\phi)$ are matrices in the Lie algebra of $G$, and the factor of $1 / 4 \pi$ has been included by convention. As in the abelian case, it is not possible for this potential to be well-defined for all $\theta$ and we will once again end up with a Dirac string. Choosing this to lie along the negative $z$-axis, we require that $\mathbf{A}_{\phi}$ vanishes at $\theta=0$ to avoid another singularity at the north pole. This necessitates that

$$
\mathbf{M}(\phi)=-\frac{\mathbf{Q}_{M}(\phi)}{4 \pi} .
$$

Utilising eqs. (3.20) and (3.21) in the second equation of motion, eq. (3.18), yields

$$
\partial_{\phi} \mathbf{Q}_{M}(\phi)=0
$$

and hence $\mathbf{Q}_{M}$ is a constant matrix. The general solution for the gauge field is therefore

$$
\mathbf{A}_{\phi}=\frac{\mathbf{Q}_{M}}{4 \pi}(\cos \theta-1) .
$$

The importance of this from a double copy perspective is that it is precisely of the form of eq. (2.7). Thus, faced with a point-like magnetic monopole in an arbitrary gauge group, one can always choose a gauge such that the double copy is straightforward, and will lead to a pure NUT charge in gravity.

\subsubsection{Allowed magnetic charges}

The gauge field of eq. (3.23) is defined with a Dirac string aligned along the negative $z$-axis. We may define this to be a "northern" gauge field $\mathbf{A}_{\mu}^{N}$ by analogy with the abelian case, and define a second potential whose only non-zero component is

$$
\mathbf{A}_{\phi}^{S}=\frac{\mathbf{Q}_{M}}{4 \pi}(\cos \theta+1)
$$

for use in the southern hemisphere. These two gauge fields are related by a non-abelian gauge transformation

$$
\mathbf{A}_{\mu}^{N}=\mathbf{S}(\varphi) \mathbf{A}_{\mu}^{S} \mathbf{S}^{-1}(\varphi)-\frac{i}{g} \mathbf{S}(\varphi) \partial_{\mu} \mathbf{S}^{-1}(\varphi)
$$

where

$$
\mathbf{S}(\varphi)=\exp \left[\frac{i g \mathbf{Q}_{M} \varphi}{2 \pi}\right]
$$

Similarly to section 3.1, we may impose the single-valuedness condition,

$$
\mathbf{S}(0)=\mathbf{S}(2 \pi),
$$

which leads to a generalised form of the Dirac quantisation condition:

$$
e^{i g \mathbf{Q}_{M}}=\mathbf{I},
$$


where I denotes the identity element in $G$. To analyse this condition further, one may write the magnetic charge matrix as a linear combination of the generators $\mathbf{H}_{i}$ of the Cartan subalgebra of $G$ [96]:

$$
\mathbf{Q}_{M}=4 \pi \mathbf{k} \cdot \mathbf{H}
$$

where $\mathbf{k}=\left(k_{1}, \ldots, k_{r}\right)$ is known as a magnetic weight vector. Taking this form of the charge matrix in the generalised quantisation condition, eq. (3.27), results in

$$
\mathbf{k} \cdot \mathbf{w}=\frac{n}{2 g}, \quad n \in \mathbb{Z},
$$

where $\mathbf{w}$ is a weight vector of the representation in which the Cartan generators are expressed. Classifying the possible monopoles in an arbitrary gauge group $G$ hence reduces to determining which magnetic weight vectors correspond to physically distinct and stable monopoles. The magnetic weights are the weights of a Lie group $G^{*}$ that is dual to $G$. To see this, recall that roots $\boldsymbol{\alpha}$ and weights w must always satisfy ${ }^{6}$

$$
\frac{2 \mathbf{w} \cdot \boldsymbol{\alpha}}{\boldsymbol{\alpha}^{2}}=N, \quad N \in \mathbb{Z}
$$

Hence, a possible solution to eq. (3.29) is to take $\mathbf{k}$ as an element of the root lattice of $G^{*}$. Recall that the root lattice of a Lie group is the sublattice of the group's weight lattice which contains the root vectors themselves. Thus, the constraint in eq. (3.29) is satisfied for

$$
\mathbf{k}=\sum_{i} n_{i} \boldsymbol{\alpha}^{(i) *}=\sum_{i} n_{i} \frac{\boldsymbol{\alpha}^{(i)}}{\left|\boldsymbol{\alpha}^{(i)}\right|^{2}}
$$

where $n_{i}$ are integers and $\boldsymbol{\alpha}^{*}=\boldsymbol{\alpha} /|\boldsymbol{\alpha}|^{2}$ are the roots of the dual group $G^{*}$. We can therefore consider two systems of roots and weights classifying two separate gauge groups, which, following the terminology of ref. [96], we refer to as the electric gauge group $G$ and the magnetic gauge group $G^{*}$. The electric group $G$ has roots $\boldsymbol{\alpha}$ and weights $\mathbf{w}$, while the magnetic group $G^{*}$ has roots $\boldsymbol{\alpha}^{*}$ and weights $\mathbf{k}$. The electric weights $\mathbf{w}$ are fixed by the fields present in the theory, and the magnetic weights $\mathbf{k}$ represent the possible magnetic charges. If both groups share the same Lie algebra, then their root vectors will differ only by a rescaling. Furthermore, if $G$ is the universal covering of the algebra then all possible magnetic weights are specified by eq. (3.31). In general more solutions will exist. Further examples of electric gauge groups $G$ and their magnetic duals $G^{*}$ can be found in ref. [96].

Naïvely, one would expect an infinite number of possible magnetic charges, corresponding to arbitrary weights in the dual weight lattice. However, not all of these correspond to physically distinct or allowable magnetic monopoles. For example, one may show that magnetic charges associated with weights $\mathbf{k}$ and $\mathbf{w}_{\mathbf{k}^{\prime}}(\mathbf{k})$ are gauge-equivalent, where

$$
\mathbf{w}_{\mathbf{k}^{\prime}}(\mathbf{k})=\mathbf{k}-2 \mathbf{k}^{\prime} \frac{\mathbf{k} \cdot \mathbf{k}^{\prime}}{\mathbf{k}^{\prime} \cdot \mathbf{k}^{\prime}}
$$

corresponds to a Weyl reflection in the hyperplane perpendicular to a third weight $\mathbf{k}^{\prime}$. After factoring out Weyl reflections, the allowed magnetic weights may still live in a number of

\footnotetext{
${ }^{6}$ For useful reviews of group theory relevant for the present context, see e.g. refs. [94, 97].
} 
different sublattices. Weights which lie within the same sublattice can be connected by an integral sum of roots, while weights in different sublattices cannot. It can be shown that monopoles with higher values of magnetic charge are dynamically unstable, such that they always decay to configurations possessing the minimum value of $\operatorname{tr}\left(Q_{M}^{2}\right)$ within each sublattice [98]. As the origin of the weight lattice corresponds to the zero magnetic charge configuration, this implies that all monopoles defined by magnetic weights within the same sublattice as the origin will decay to the vacuum state.

It is important to note that this instability of monopoles with non-minimal magnetic weights within a given sublattice necessitates a careful specification of the gauge groups. If either the electric or magnetic gauge group is the universal covering group $\tilde{G}$ of the Lie algebra, then the other will be the adjoint group $\tilde{G} / K$, where $K$ is the centre of $\tilde{G}$. Thus if we consider Yang-Mills with $G=\mathrm{SU}(N)$, the magnetic group will be $G^{*}=\mathrm{SU}(N) / \mathbb{Z}_{N}$. However, all weights of $\mathrm{SU}(N) / \mathbb{Z}_{N}$ lie in the same sublattice as the weight at the origin of the lattice. All monopole solutions for $G=\mathrm{SU}(N)$ are therefore dynamically unstable and will reduce to the vacuum solution. This, however, is not the case for $G=\mathrm{SU}(N) / \mathbb{Z}_{N}$ and $G^{*}=\mathrm{SU}(N)$, as the $N$ sublattices of the weight lattice of $\mathrm{SU}(N)$ allow for $N-1$ stable monopole solutions. This has an elegant topological interpretation that is directly related to the abelian case, as we now describe.

\subsubsection{Topology of the non-abelian monopole}

In section 3.1, we reviewed the Wu-Yang fibre bundle interpretation of the Dirac monopole, in which two gauge fields are patched together, thus generating a gauge configuration with non-trivial topology. In this section, we describe the generalisation of this picture to the non-abelian case [93]. We are again considering singular monopoles, so that the origin of spacetime is removed, and spatial slices are then topologically equivalent to $S^{2}$. This in turn necessitates at least two coordinate patches, which we may again choose to be the northern and southern hemispheres of figure 2(b). Similarly, we may also construct the family of curves of figure 3 , each of which can be associated with an element of the gauge group,

$$
\mathbf{U}(\theta)=\mathcal{P} \exp \left[i g \oint_{C(\theta)} d x^{\mu} \mathbf{A}_{\mu}\right],
$$

where $\mathcal{P}$ denotes path ordering of the (non-commuting) gauge fields $\mathbf{A}_{\mu}$ along the contour. As $\theta$ varies from 0 to $\pi$, this traces out a curve in the electric gauge group $G$, where the points $\theta=0, \pi$ both correspond to the identity element. At $\theta=\pi / 2$, the gauge field switches from its northern to southern form, leading once again to the situation shown in figure 3(b), where $A$ and $B$ must correspond to equivalent group elements. Denoting the group of such transformations between $A$ and $B$ by $H$, one may write the general patching condition between the northern and southern gauge fields as

$$
\mathcal{P} \exp \left[i g \oint_{C} d x^{\mu} \mathbf{A}_{\mu}^{N}\right]=\mathbf{U}_{H}\left\{\mathcal{P} \exp \left[i g \oint_{C} d x^{\mu} \mathbf{A}_{\mu}^{S}\right]\right\}
$$

where $\mathbf{U}_{H}$ constitutes an element of $H$, and $C \equiv C(\pi / 2)$ is the equator of the sphere in figure $3(\mathrm{a})$. This is the general condition that encodes the non-trivial topology of the 
singular non-abelian monopole. As in the abelian case, this non-trivial topology is classified by the first homotopy group of the electric gauge group $\pi_{1}(G)$, which follows directly from the fact that $\pi_{1}(G)$ characterises the different ways in which one may join the points $A$ and $B$ in figure 3(b) to form a closed curve. As an example, we may take an electric group $G=\tilde{G} / K$, in which case $B$ can be related to $A$ by any element of $K$, the centre of the universal covering $\tilde{G}$. The relevant first homotopy group is then

$$
\pi_{1}(\tilde{G} / K)=K
$$

Thus for $G=\mathrm{SU}(N) / \mathbb{Z}_{N}$, we find $\pi_{1}(G)=\mathbb{Z}_{N}$. The identity element corresponds to monopole configurations that are topologically equivalent to the vacuum, and hence unstable. This leaves $N-1$ stable monopoles, consistent with the discussion of the previous section.

Note that eq. (3.34) reduces to eq. (3.9) for an abelian electric group $G^{*}=\mathrm{U}(1)$. A general element of $H$ in then given by

$$
\mathbf{U}_{H}=e^{2 \pi i n}, \quad n \in \mathbb{Z},
$$

from which eq. (3.9) follows. This can in turn be related to eq. (3.4), which is proportional to the first Chern number that classifies the non-trivial topology of $\mathrm{U}(1)$ principal bundles. For other gauge groups, however, the patching condition will not be relatable to the same topological invariant. For example, if one replaces the gauge group $\mathrm{U}(1)$ with $\mathrm{SU}(N)$, the first Chern number vanishes (due to tracelessness of the generators), but one can instead classify the topology of solutions using what we may refer to as the Woodward classes of ref. [99], as we discuss in appendix A. The relevance for the double copy is as follows. Previous studies [86] have suggested that given characteristic classes in gauge theories may be related to similar quantities in gravity theories, under the double copy. This creates a puzzle, in that characteristic classes depend upon the gauge group, whereas colour information is irrelevant for the gravity side of the double copy. Here we see that there is in fact no problem, as it is simply not true that the double copy should apply to individual characteristic classes. The appropriate quantity that classifies the "double copiable" topology of the gauge theory solution is instead the patching condition of eq. (3.34), whose form is independent of the gauge group. We will see in the following section that this indeed has a gravitational counterpart.

\section{Topology of the Taub-NUT solution}

The Taub-NUT solution of refs. [87, 88] is a widely studied exact solution of GR, whose gravitational field has a rotational character that does not die off at spatial infinity. As discussed in section 2, the general Taub-NUT solution has a Schwarzschild-like mass term $M$, and an additional NUT charge $N$, where it is the latter that gives rise to the rotational aspect of the field. Analogies between magnetic monopoles in non-abelian gauge theories and the Taub-NUT solution have been made many times before (see e.g. ref. [100] for a review). However, ref. [45] formalised this by pointing out that the classical double copy 
implies an exact relationship between dyons and Taub-NUT, which is true for all values of the radial coordinate. ${ }^{7}$ The double copy is manifest in a particular coordinate system in $(2,2)$ signature [92], such that the metric assumes the double Kerr-Schild form of eq. (2.5). However, once the double copy between solutions is known, we may make a coordinate transformation to more conventional coordinates. To this end, it is convenient to work with the Taub-NUT line element in spherical polar coordinates,

$$
d s^{2}=f(r)[d t+2 N(\cos \theta-1) d \varphi]^{2}-f^{-1}(r) d r^{2}-\left(r^{2}+N^{2}\right) d \Omega^{2},
$$

where

$$
d \Omega^{2}=d \theta^{2}+\sin ^{2} \theta d \varphi^{2}
$$

is the squared element of solid angle in four spacetime dimensions, and the additional quantities are given by

$$
f(r)=\frac{\left(r-r_{+}\right)\left(r-r_{-}\right)}{r^{2}+N^{2}}, \quad r_{ \pm}=M \pm \sqrt{M^{2}+N^{2}} .
$$

Here $M$ and $N$ are the Schwarzschild-like mass and NUT charge parameters of eq. (2.5) respectively. The case of a pure NUT charge is given by the limit $M \rightarrow 0$, in which case eq. (4.1) reduces to

$$
d s^{2}=\frac{r^{2}-(\kappa N)^{2}}{r^{2}+(\kappa N)^{2}}[d t+2 \kappa N(\cos \theta-1) d \varphi]^{2}-\left(r^{2}+(\kappa N)^{2}\right)\left[\frac{d r^{2}}{r^{2}-(\kappa N)^{2}}+d \Omega^{2}\right],
$$

where we have rescaled $N \rightarrow \kappa N$ for later convenience. The metric has a coordinate singularity at $\theta=\pi$. This is the so-called Misner string [103], and is a direct analogue of the Dirac string for a magnetic monopole. The known solution to this problem is that one may use two different coordinate patches, obtained by splitting spatial slices into a northern and southern hemisphere, as in figure 2. In the northern hemisphere $(\theta \leq \pi / 2)$, the metric of eq. (4.4) is well-defined, and one regards $t$ as a "northern" time coordinate $t \equiv t_{N}$. In the southern hemisphere, one may transform to a "southern" time coordinate

$$
t_{N}=t_{S}+4 \kappa N \varphi
$$

such that the northern and southern line elements are given by

$$
d s_{N, S}^{2}=\frac{r^{2}-(\kappa N)^{2}}{r^{2}+(\kappa N)^{2}}\left[d t_{N, S}+2 \kappa N(\cos \theta \mp 1) d \varphi\right]^{2}-\left(r^{2}+(\kappa N)^{2}\right)\left[\frac{d r^{2}}{r^{2}-(\kappa N)^{2}}+d \Omega^{2}\right],
$$

where the upper (lower) sign corresponds to the northern (southern) case respectively. One sees that the southern metric is singular at $\theta=0$, and thus that the Misner string has been moved into the northern hemisphere. Given that the azimuthal coordinate lies in the range $0 \leq \varphi<2 \pi$, eq. (4.5) (which applies on the equator on which the two coordinate patches overlap) then implies that both $t_{N}$ and $t_{S}$ must be periodic with period

$$
t_{0}=8 \pi \kappa N_{0},
$$

\footnotetext{
${ }^{7}$ The Taub-NUT solution has also been used to examine the double copy of the electromagnetic duality symmetry that operates in gauge theories [79, 101, 102].
} 
where $N_{0}$ is a basic unit of NUT charge. Indeed, it is known that this property is required for the spacetime to be fully spherically symmetric [104, 105]. The full NUT charge appearing in eq. (4.6) is then given by

$$
N=n N_{0}, \quad n \in \mathbb{Z},
$$

where the arbitrary integer $n$ corresponds to the fact that the transformation between $t_{S}$ and $t_{N}$ is defined only up to an integer multiple of the time period $t_{0}{ }^{8}$

From the double copy perspective, it is desirable to obtain the above periodicity condition from a procedure that makes its relationship with the gauge theory manifest. We may carry this out as follows. First, a standard result in GR is that there is a time holonomy upon trying to synchronise clocks around a closed contour. That is, upon traversing a given closed loop $C$, the difference in time coordinate upon returning to the starting point satisfies [106]

$$
|\Delta t|=\oint_{C} \frac{g_{0 i}}{g_{00}} d x^{i}
$$

where $i \in\{1,2,3\}$ is a spatial index, and the lack of manifest Lorentz covariance on the right-hand side follows from the explicit choice of the physical time coordinate on the lefthand side. This takes a suggestive form if one considers asymptotically large distances $r \rightarrow \infty$, such that the metrics arising from eq. (4.6) take the form

$$
g_{\mu \nu}^{N, S}=\eta_{\mu \nu}+\kappa h_{\mu \nu}^{N, S}
$$

where the only non-zero components of the northern and southern gravitons are given by

$$
h_{0 \varphi}^{N, S}=2 N(\cos \theta \mp 1), \quad h_{\varphi \varphi}^{N, S}=4 \kappa N^{2}(\cos \theta \mp 1)^{2} .
$$

Then the time holonomy of eq. (4.9) can be written purely in terms of the graviton field, as

$$
|\Delta t|=\kappa \oint_{C} h_{0 i} d x^{i}
$$

Let us now consider the family of curves $C(\theta)$ of figure $3(\mathrm{a})$, consisting of circles on the 2 -sphere at spatial infinity. One may associate each such curve with a value of the time holonomy in eq. (4.12), which from eqs. (4.11) yields

$$
\left|\Delta t^{N, S}(\theta)\right|=\kappa \oint_{C} h_{0 \varphi}^{N, S} d \varphi=2 \pi \kappa h_{0 \varphi}^{N, S}
$$

where we have used the fact that the components $\left\{h_{0 \varphi}^{N, S}\right\}$ are independent of $\varphi$. The time shifts of eq. (4.13) form a subgroup of the general group of diffeomorphisms that act in gravity. One may thus view eq. (4.13) as forming a map from the set of curves in figure $3(\mathrm{a})$ to the diffeomorphism group. At $\theta=0$, the loop $C(0)$ is infinitely small, such that $\Delta t=0$, which constitutes the identity element of the group. As $\theta$ increases, a path is traced out in the diffeomorphism group until the equator $\theta=\pi / 2$ is reached. At this

\footnotetext{
${ }^{8}$ From eq. (4.7), one may either take $N_{0}$ as a free parameter, in terms of which $t_{0}$ is fixed, or vice versa. This is analogous to the quantisation condition between the electric and magnetic charges in abelian gauge theory.
} 
point, the graviton field jumps from its northern to its southern form. Finally, as $\theta \rightarrow \pi$, the curve $C(\theta)$ becomes infinitely small again, and we are back at the identity element. This is precisely the picture of figure 3(b), that we have previously used for elements of a (non-)abelian gauge group. In order for the gravitons to be patched on the equator i.e. to correspond to the same physical solution, the time holonomies arising from $h_{\mu \nu}^{N}$ and $h_{\mu \nu}^{S}$ must be physically equivalent. However, substituting the explicit results of eqs. (4.11) into eq. (4.13) yields

$$
\left|\Delta t^{S}(\pi / 2)\right|-\left|\Delta t^{N}(\pi / 2)\right|=8 \pi \kappa N .
$$

The only way that the time holonomies can coincide is then to impose periodicity of $t^{N, S}$ with period $t_{0}$ as in eq. (4.7).

There is another way to reach the same conclusion, that makes contact with Dirac's original argument for the quantisation of electric charge in an abelian gauge theory [107]. Reference [108] considered a gravitational analogue of the magnetic monopole, by considering the phase experienced by a non-relativistic test particle of mass $m$ that traverses a given loop $C$ in space, and finding (if $h_{00}=0$ ) that it is given in the weak field limit by

$$
\Phi=\exp \left[i \kappa m \oint_{C} d x^{i} h_{0 i}\right]
$$

which is directly related to eq. (4.12). For this phase to be well-defined on the equator of figure 3 where the two coordinate patches overlap, one must have that the difference in phases evaluated with the northern and southern graviton fields is a multiple of $2 \pi$, such that

$$
\kappa m \oint_{C} d x^{i}\left[h_{0 i}^{N}-h_{0 i}^{S}\right]=2 \pi n, \quad n \in \mathbb{Z} .
$$

Substituting the results of eqs. (4.11) yields

$$
m \kappa N=\frac{n}{4},
$$

which says that the mass $m$ entering the phase of eq. (4.15) is quantised. Reference [108] was unsure about what to take for this mass. However, its interpretation becomes clear given the discussion above regarding the periodicity of the time coordinate. The mass $m$ refers to the energy of a static wavefunction in the presence of the NUT charge. If the time coordinate is compact with period $t_{0}$, this implies that the mass $m$ is quantised according to

$$
\Delta m=\frac{2 \pi}{t_{0}} .
$$

Equation (4.17) implies

$$
\Delta m=\frac{1}{4 \kappa N_{0}},
$$

so that combining this with eq. (4.18) yields eq. (4.7) as required.

The description of the quantisation condition in terms of the gravitational phase experienced by a test particle allows one to write down a patching condition for Taub-NUT that is directly analogous to the gauge theory condition of eq. (3.34). First, note that the phase around a loop defines a map from the base spacetime to the group $\mathrm{U}(1)$, so that the curves 
of figure 3(a) lead to the description in figure 3(b), where the latter is now interpreted as a path in the group manifold of $\mathrm{U}(1)$. The requirement that the phases for the northern and southern fields are related by a multiple of $2 \pi$ can be written as

$$
\exp \left[i \kappa m \oint_{C} d x^{\mu} h_{0 \mu}^{N}\right]=U_{H} \exp \left[i \kappa m \oint_{C} d x^{\mu} h_{0 \mu}^{S}\right]
$$

where we have used the fact that $h_{00}=0$, and $U_{H}$ is an element of the group of transformations that leaves the phase invariant, and which thus connects the points $A$ and $B$ in figure 3(b). A general such element is written in eq. (3.35), and this description makes clear that the topology of the Taub-NUT solution is similar to the case of an abelian gauge theory. That is, the non-trivial topology is classified by maps from the equator at infinity to $\mathrm{U}(1)$, namely by the first homotopy group of eq. (3.8). A similar conclusion was reached by ref. [89], which formally defined the NUT charge according to the integral ${ }^{9}$

$$
N=\frac{\kappa}{8 \pi} \iint_{S} \partial_{i} h_{0 j} d x^{i} \wedge d x^{j}
$$

where $S$ is the 2 -sphere at spatial infinity. This can be verified by separating the field into northern and southern parts related by the time translations of eq. (4.5), before applying Stokes' theorem and using the results of eqs. (4.11). The full NUT charge is given by an integer multiple of the basic unit $N_{0}$, according to eq. (4.8). Indeed, interpreting the integrand of eq. (4.21) as a field strength, one finds that $N$ is proportional to the first Chern number, that specifies the non-trivial topology of a U(1) bundle [89]. The Chern number is integer-valued, and thus amounts to $n$ in the above construction. Furthermore, the $\mathrm{U}(1)$ fibres in this case correspond to the group of time translations with a periodic time coordinate, rather than the group of phases experienced by particles moving along a path. However, the pictures are ultimately equivalent given that the phase experienced by a non-relativistic particle is governed directly by the Hamiltonian, which is conjugate to the time variable.

Above, we have seen that one may describe the pure NUT charge in gravity using one of two descriptions, both of which are equivalent. That is, one may divide space into two hemispheres and define the graviton field differently in each, such that the definitions are related by a diffeomorphism. In the weak field and non-relativistic limit, one may write a patching condition - eq. (4.20) - that is the precise analogue of its counterpart in (abelian) gauge theory, and which characterises the non-trivial topology of the graviton field. The ingredients we have used to formulate the patching condition of eq. (4.20) are not new. However, they have not previously been analysed from the point of view of the double copy. The fact that eq. (4.20) is a precise counterpart of eq. (3.34) tells us that it is indeed possible to map global information from gauge theory to gravity under the double copy, such that the latter becomes more than merely a local statement. Furthermore, it should be no surprise that the patching condition on the gravity side takes an abelian-like form, given that colour structure is stripped off upon taking the double copy. The patching

\footnotetext{
${ }^{9}$ Our definition differs slightly to that of ref. [89] due to our explicit inclusion of factors of $\kappa$.
} 
conditions of eqs. (3.34) and (4.20) then form an explicit realisation of figure 1, applied to global properties of exact solutions.

It is possible to relate the gauge theory and gravity patching conditions to previous work in the context of scattering amplitudes, which we shall do in the following section. Before moving on, however, it is worth drawing attention to the recent study of ref. [109], which provides an alternative way to describe the Taub-NUT solution by patching together graviton fields analogously to the Wu-Yang construction [93]. Instead of relating the northern and southern fields by a diffeomorphism, the authors instead use a BMS dual supertranslation [110,111], which is defined only at asymptotic infinity, and thus not formally equivalent to a diffeomorphism. They then argue that a periodic time coordinate is no longer needed, which avoids the well-known problem that the Taub-NUT metric gives rise to pathological closed time-like curves. There are similarities between ref. [109] and the analysis carried out here, most notably the use of a Wu-Yang-like formulation for the graviton patching, and also the fact that our patching condition of eq. (4.20) is defined in the weak field limit, and thus at asymptotic infinity. However, there is a crucial difference between our ethos and that of ref. [109]: the double copy for the Taub-NUT solution applies to the entire classical solutions [45], not just at asymptotic infinity. Furthermore, the fact that gauge transformations on the gauge theory side of the correspondence should be associated with diffeomorphisms on the gravity side is well-known in the double copy literature on both exact classical solutions and scattering amplitudes. We have thus used the traditional formulation of Taub-NUT in this paper, but stress that the relevance (or otherwise) of ref. [109] deserves further study. It is also worth emphasising that the results of ref. [109] are useful for the study of Taub-NUT metrics, independently of the double copy.

\section{Wilson lines and the double copy}

In the previous sections, we have seen that the non-trivial topology of a (non)-abelian magnetic monopole can be written in terms of a certain patching condition - eq. (3.34) - that involves the gauge-covariant phase experienced by a particle moving around the equator of the 2-sphere at infinity. This corresponds to a so-called Wilson line operator, or a Wilson loop in this case due to the closed nature of the contour. Such operators appear in many places in the study of quantum field theory, arising whenever gauge-dependent information must be compared at different points in spacetime. What is perhaps less wellknown is that Wilson lines have also been defined in gravity: see e.g. refs. [112-116] for (in some cases very) early works on this subject. In recent times, gravitational Wilson lines have been used to try to set up common languages between non-abelian gauge theories and gravity $[28-30,117,118]$, which is particularly convenient from a double copy point of view. In this section, we point out how the results of the previous section can be expressed in terms of Wilson lines, and how this relates to other examples of the double copy in the literature.

Motivated by the gauge theory case, we define a gravitational Wilson line in terms of the phase experienced by a scalar test particle in a gravitational field. In the full covariant 
theory, this must be proportional to the proper length of a given path $C$, so that we define

$$
\Phi_{\text {grav. }}(C)=\exp \left[i m \int_{C} d s\left(g_{\mu \nu} \frac{d x^{\mu}}{d s} \frac{d x^{\nu}}{d s}\right)^{1 / 2}\right]
$$

where $x^{\mu}(s)$ is a parametrisation of the curve, and $m$ the mass of the test particle. This expression simplifies in the weak field limit. Writing

$$
g_{\mu \nu}=\eta_{\mu \nu}+\kappa h_{\mu \nu}
$$

one may expand eq. (5.1) in $\kappa$ to obtain

$$
\Phi_{\text {grav. }}(C)=\exp \left[\frac{i \kappa}{2} \int_{C} d s \frac{d x^{\mu}}{d s} \frac{d x^{\nu}}{d s} h_{\mu \nu}(x)\right],
$$

where we have ignored an overall normalisation constant that is independent of $h_{\mu \nu}$ (and that will cancel in any normalised expectation value of Wilson line operators). We have also absorbed the mass $m$ into the length parameter $s$. Equation (5.2) is the Wilson line operator that was considered in e.g. refs. [28-30, 115, 117], where it was used to analyse properties of scattering amplitudes. ${ }^{10}$ Here we may make contact with eq. (4.15) by taking $C$ to be the equator of the 2 -sphere at infinity, and taking $s=m t$, where $t$ is the conventional time coordinate:

$$
\Phi_{\text {grav. }}(C)=\exp \left[\frac{i \kappa m}{2} \oint_{C} d t\left(h_{00}+2 \dot{x}^{i} h_{0 i}+\dot{x}^{i} \dot{x}^{j} h_{i j}\right)\right],
$$

where the dot represents differentiation with respect to $t$. For the Taub-NUT solution considered in the previous section, $h_{00}=0$. Then, in the non-relativistic (small velocity) limit, eq. (5.3) reduces to eq. (4.15) as required.

There is a very well-defined sense in which the gravitational Wilson line operator of eq. (5.2) is a double copy of its gauge theory counterpart

$$
\Phi(C)=\mathcal{P} \exp \left[i g \int_{C} d s \frac{d x^{\mu}}{d s} \mathbf{T}^{a} A_{\mu}^{a}(x)\right],
$$

where $\mathbf{T}^{a}$ is a generator of the gauge group in the appropriate representation (n.b. this is absorbed into the gauge field in eq. (3.34)), and $s$ a parameter along the curve with the same mass dimension as in eq. (5.2). To go from eq. (5.4) to eq. (5.2), one must replace the coupling constant with its gravitational counterpart:

$$
g \rightarrow \frac{\kappa}{2}
$$

which is precisely the BCJ prescription for scattering amplitudes [3]. Furthermore, one must also strip off the colour generator $\mathbf{T}^{a}$, and replace this with a second kinematic factor representing the tangent vector to the Wilson line contour:

$$
\mathbf{T}^{a} \rightarrow \frac{d x^{\nu}}{d s} .
$$

\footnotetext{
${ }^{10}$ Although we considered massive particles above, eq. (5.2) can be generalised for massless particles. In that case, one must replace the exponent in eq. (5.1) with the action for a massless point particle, involving an einbein (see e.g. ref. [119]).
} 
This mirrors the replacement of colour information by kinematics in the scattering amplitude double copy. Indeed, there are existing cases of the amplitude double copy that can be entirely expressed in terms of Wilson lines, such that the underlying mechanism of the double copy is precisely the replacements made above. This has not necessarily been realised in the existing literature, and so it is worthwhile to briefly review these examples.

\subsection{The all-order structure of infrared singularities}

If one dresses an $n$-point scattering amplitude with virtual gluon or graviton radiation, one encounters infrared (IR) divergences associated with the exchanged gauge bosons becoming "soft" (i.e. having vanishing 4-momenta). ${ }^{11}$ These singularities have a universal form that factors off from the full scattering amplitude $\mathcal{A}$, which has a simple physical interpretation: soft radiation has an infinite Compton wavelength, and thus cannot resolve the underlying interaction that produced the $n$ hard particles. One may thus write (see e.g. ref. [123] for a pedagogical review)

$$
\mathcal{A}=\mathcal{H} \cdot \mathcal{S},
$$

where $\mathcal{H}$ is a so-called hard function that is completely finite, and $\mathcal{S}$ a soft function that collects all the soft singularities. The latter is known to have an exponential form, where calculating additional terms in the logarithm of the soft function amounts to summing up infrared singularities to all orders in perturbation theory. The structure of the soft function in QCD and QED is only partially known ${ }^{12}$ (see e.g. ref. [124]), and that of gravity is known exactly. That is, it has now been well-established that the logarithm of the soft function in gravity terminates at first order in the gravitational coupling constant [117, 121].

Reference [27] used the above properties to present all-loop order evidence for the BCJ double copy. That is, the authors showed that one may isolate IR singularities at any given order in perturbation theory in either QED or QCD, and double copy them to obtain the known IR singularities of gravity. The somewhat lengthy analysis used intricate Feynman-diagrammatic arguments, making clear how the BCJ duality between colour and kinematics could be satisified at arbitrary loop orders, if terms outside of the soft limit could be neglected. Furthermore, the authors noted that the IR singluarities of either QED or QCD both mapped to the same gravitational results, thus providing an explicit realisation of figure 1 .

Here, we wish to point out that the analysis of ref. [27] would have been drastically simpler using Wilson lines. It is known in (non)-abelian gauge theories that the soft function can be expressed as a vacuum expectation value of Wilson line operators, whose contours correspond to the physical trajectories

$$
x_{i}^{\mu}=s p_{i}^{\mu}
$$

of the outgoing hard particles (see e.g. ref. [124]):

$$
\mathcal{S}=\left\langle 0\left|\prod_{i} \Phi_{i}\right| 0\right\rangle, \quad \Phi_{i} \equiv \mathcal{P} \exp \left[i g \mathbf{T}^{a} p_{i}^{\mu} \int_{0}^{\infty} d s A_{\mu}^{a}\right] .
$$

\footnotetext{
${ }^{11}$ In (non)-abelian gauge theories, one also encounters singularities when radiation is collinear with the external particles in the interaction, although such singularities are absent in gravity [117, 120-122].

${ }^{12}$ Interestingly, the soft function of QED is known exactly if there are no propagating fermions.
} 
The physics of this result is that hard particles emitting soft radiation cannot recoil, and thus can only change by a phase. For this phase to have the right gauge covariance properties to form part of a scattering amplitude, it can only be a Wilson line. Armed with the gravitational Wilson line operator of eq. (5.2), we might write a similar definition in gravity [117]:

$$
\mathcal{S}_{\text {grav. }}=\left\langle 0\left|\prod_{i} \Phi_{\text {grav. }, i}\right| 0\right\rangle, \quad \Phi_{\text {grav. }, i} \equiv \exp \left[\frac{i \kappa}{2} p_{i}^{\mu} p_{i}^{\nu} \int_{0}^{\infty} d s h_{\mu \nu}\right] .
$$

The all-order double copy of IR singularities obtained using diagrammatic arguments then follows simply from the observations of eqs. (5.5), (5.6), namely that the Wilson line exponents themselves double copy in a precise way. Furthermore, the fact that both abelian and non-abelian results both reproduce the same gravity result is obvious; one strips off the colour generator $\mathbf{T}^{a}$ in eq. (5.8), so that it does not matter if one starts with a QED or QCD Wilson line.

There is potentially a rather subtle flaw in the above argument, namely that for VEVs of Wilson lines to double copy in arbitrary circumstances, the propagator for the graviton must be written in a form which manifestly decouples left and right-indices. This is related to the fact [125] that the double copy of pure gauge theory is not pure gravity, but a theory containing a dilaton and axion (two-form) field. In any case, the axion and dilaton do not pose a problem: both are scalar degrees of freedom in four spacetime dimensions, and by standard power-counting arguments will not contribute to the structure of leading IR singularities at each order in perturbation theory [27]. Going beyond the leading soft approximation, one may indeed be sensitive to the additional matter content (see e.g. [64] for an interesting discussion of this point).

\subsection{The Regge limit}

Another kinematic limit of scattering amplitudes in which all-order information is obtainable is the high-energy or Regge limit of $2 \rightarrow 2$ scattering, which can be expressed (for massless particles) as

$$
s \gg-t, \quad s=\left(p_{1}+p_{2}\right)^{2}, \quad t=\left(p_{1}-p_{3}\right)^{2},
$$

where we have labelled 4-momenta as in figure 4(a), and the Mandelstam invariants $s$ and $t$ constitute the squared centre of mass energy and momentum transfer respectively. In the Regge limit of eq. (5.10), the particles suffer a very small deflection owing to the small momentum transfer. Thus, in position space, they follow approximately straightline trajectories, such that the outgoing particles become approximately collinear with the incoming ones i.e.

$$
p_{3} \simeq p_{1}, \quad p_{4} \simeq p_{2} .
$$

The lack of recoil means that the particles can only change by a phase and, using similar arguments to the previous section, we arrive at the idea that $2 \rightarrow 2$ scattering in the Regge limit can be described (in position space) by a vacuum expectation value of Wilson lines 


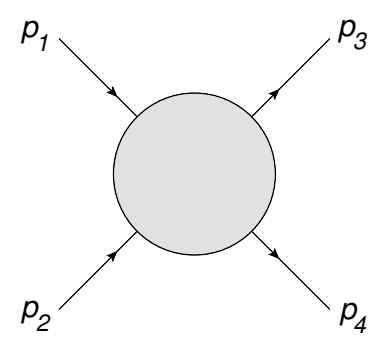

(a)

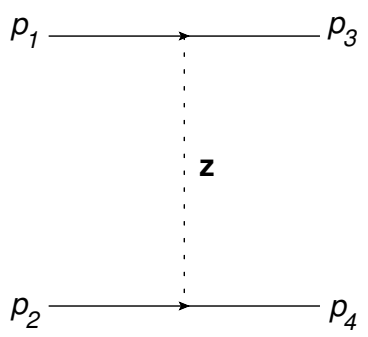

(b)

Figure 4. (a) Momentum labels for $2 \rightarrow 2$ scattering; (b) In the Regge limit, one may model the scattering by two Wilson lines separated by a transverse impact parameter $\boldsymbol{z}$.

separated by a transverse displacement $\boldsymbol{z}$, where the magnitude of the latter is the impact parameter, or distance of closest approach:

$$
\mathcal{A}_{s \gg|t|} \rightarrow\left\langle 0\left|\Phi\left(p_{1}, 0\right) \Phi\left(p_{2}, \boldsymbol{z}\right)\right| 0\right\rangle, \quad \Phi(p, \boldsymbol{z})=\mathcal{P} \exp \left[i g_{s} p^{\mu} \int_{-\infty}^{\infty} d s A_{\mu}(s p+z)\right] .
$$

This framework was first developed in the classic work of refs. [126, 127], which showed that known properties of the Regge limit in QCD emerge from the Wilson line approach. Reference [29] demonstrated that the same picture works for gravity if the QCD Wilson lines are replaced with equivalent ones based on eq. (5.2). The Wilson line language thus makes the calculations in QCD and gravity look essentially identical, although the physics in each theory turns out to be very different. Furthermore, the Wilson line double copy reproduces previous studies of the Regge limit based on diagrammatic arguments [31-34], where the results overlap.

Above, we have seen two examples in which the Wilson line double copy replacements of eqs. (5.5), (5.6) are directly related to the BCJ double copy for scattering amplitudes. The fact that Wilson lines also underly our identification of global topological information for the magnetic monopole and NUT charge suggests that they form a useful bridge, relating different manifestations of the double copy. This also begs the question of what else can be done with gravitational Wilson lines, an issue which certainly merits further study.

\section{Conclusion}

In this paper, we have examined the double copy for exact classical solutions, which is normally expressed as a local statement relating the graviton field to products of gauge fields. We have instead considered whether it is possible to map global properties of solutions between theories, using the magnetic monopole in gauge theory as a test case. A non-trivial global structure for monopole solutions is usually characterised by topological invariants that depend on the field strength. However, these invariants can be different for different gauge groups, which makes it difficult to see how to associate this information with a unique gravity counterpart.

To clarify these issues, we first reviewed how to express a general singular monopole solution for arbitrary gauge groups. By a suitable gauge choice, this can always be expressed 
as a constant colour matrix multiplying an abelian-like monopole field, so that the double copy to gravity is straightforward: one simply removes the colour matrix, and replaces the monopole with a pure NUT charge, as follows from ref. [45]. This provides an explicit realisation of figure 1 for exact classical solutions, and extends the recent $\mathrm{SU}(2)$ results of ref. [85] to arbitrary gauge groups.

We then reviewed the Wu-Yang fibre bundle construction for magnetic monopoles [93], in which one defines separate gauge fields associated with the northern and southern spatial hemispheres. These are patched together on the equator, to create a global field that is free of string-like singularities, but topologically non-trivial. The topology is classified by the patching condition, which can always be expressed in terms of Wilson lines representing the phase experienced by a particle upon being taken around the equator (eq. (3.34)). Next, we showed that a similar construction can be applied in gravity (see also the recent ref. [109]), using the known time translations that remove the Misner string singularity in each coordinate patch [103], and which result in a periodic time coordinate. This treats the NUT solution as a U(1) bundle, where one may take the fibres to correspond either to time translations (modulo the time period), or the group of phases experienced by a nonrelativistic particle being taken around a loop. The latter description maps most cleanly to the Aharonov-Bohm effect in magnetism [108], and the resulting patching condition can then be expressed in terms of gravitational Wilson lines.

As we argued in section 5, the appearance of Wilson lines makes the double copy particularly natural, given that the relevant gauge theory and gravity operators are related by simple replacements of coupling constants, and colour information by kinematics. We also saw that previous double copy properties of scattering amplitudes had neat interpretations in terms of Wilson lines. One of these (the Regge limit) was already known [29], but the other (the double copy structure of IR singularities in fixed-angle scattering [27]) has not been previously understood in this way in the literature. This strongly suggests that there are other uses of gravitational Wilson lines in a double copy context, and that they potentially have a key role to play in establishing a bridge, where appropriate, between the double copy literature on scattering amplitudes and that relating to classical solutions.

We hope that the results of this study provide useful food for thought in widening the remit of the double copy yet further, including non-perturbative and/or global aspects.

\section{Acknowledgments}

We thank Prarit Agarwal, George Barnes, David Berman, Adrian Padellaro and Costis Papageorgakis for useful discussions. This work has been supported by the U.K. Science and Technology Facilities Council (STFC) Consolidated Grant ST/P000754/1 "String theory, gauge theory and duality", and by the European Union Horizon 2020 research and innovation programme under the Marie Skłodowska-Curie grant agreement No. 764850 "SAGEX".

\section{A Topological classification of $\mathrm{SU}(N) / \mathbb{Z}_{N}$ bundles}

In section 3.2, we reviewed how the non-trivial topology of a Dirac monopole can be described by the first Chern class. In the case of other gauge groups, it is not necessarily 
the case that a well-known invariant characterises the non-trivial topology obtained by patching together gauge fields in the northern and southern spatial hemispheres. However, for $\mathrm{SU}(N)$ groups there is indeed a known description, which we thought worth pointing out in this appendix. These results were first obtained by Woodward in ref. [99].

As discussed in section 3.2, we consider magnetic solutions associated with an electric gauge group $G=\mathrm{SU}(N) / \mathbb{Z}_{N}$, otherwise known as $\mathrm{PU}(N)$. More formally, we can write the following short exact sequence relating the various groups appearing above:

$$
\mathbb{Z}_{N} \stackrel{\text { center }}{\longrightarrow} \mathrm{SU}(N) \stackrel{\text { quotient }}{\longrightarrow} \mathrm{PU}(N) \cong \mathrm{SU}(N) / \mathbb{Z}_{N} \text {. }
$$

Associated with this sequence is a corresponding exact sequence relating the following homotopy groups:

$$
\cdots \rightarrow \pi_{1}\left(\mathbb{Z}_{N}\right) \rightarrow \pi_{1}(\mathrm{SU}(N)) \rightarrow \pi_{1}(\mathrm{PU}(N)) \rightarrow \pi_{0}\left(\mathbb{Z}_{N}\right) \rightarrow \pi_{0}(\mathrm{SU}(N)) \rightarrow \pi_{0}(\mathrm{PU}(N))
$$

from which we obtain the isomorphism:

$$
\pi_{1}(\mathrm{PU}(N)) \cong \pi_{0}\left(\mathbb{Z}_{N}\right)=\mathbb{Z}_{N}
$$

The first homotopy group on the left-hand side is what classifies $\mathrm{PU}(N)$ bundles on $S^{2}$ base manifolds, as explained previously using figure 3. Such manifolds arise in our context due to the fact that all of the magnetic monopoles we consider are singular at the origin, and thus are defined on $\mathbb{R}^{3}-\{0\}$, which is homotopic to $S^{2}$. Each topologically distinct monopole solution corresponds to a distinct fibre bundle, and from eq. (A.3), we see that there are $N$ topologically different monopole solutions associated with electric group $\mathrm{PU}(N)$ each corresponding to a different element of the first homotopy group. However, we can also classify the solutions using the dual description of cohomology classes, where eq. (A.2) implies the exact sequence

$$
\cdots \rightarrow H^{1}\left(S^{2}, \mathrm{SU}(N)\right) \rightarrow H^{1}\left(S^{2}, \mathrm{PU}(N)\right) \rightarrow H^{2}\left(S^{2}, \mathbb{Z}_{N}\right) \rightarrow H^{2}\left(S^{2}, \mathrm{SU}(N)\right) \rightarrow \cdots
$$

and hence the isomorphism of cohomology groups

$$
H^{1}\left(S^{2}, \mathrm{PU}(N)\right) \cong H^{2}\left(S^{2}, \mathbb{Z}_{N}\right)=\mathbb{Z}_{N}
$$

We refer to $[\omega] \in H^{2}\left(S^{2}, \mathbb{Z}_{N}\right)$ as the 2nd Woodward class of the $\mathrm{PU}(N)$-bundle, after ref. [99]. For $N=2$ we have $\mathrm{PU}(2)=\mathrm{SO}(3)$ and the 2 nd Woodward class reduces to the 2nd Stiefel-Whitney class. In other words, the Stiefel-Whitney class replaces the first Chern class as the relevant characteristic class for monopoles in $\mathrm{SU}(2)$ gauge theory. For $\mathrm{SU}(N)$ with $N>2$, one must use the Woodward classes.

Open Access. This article is distributed under the terms of the Creative Commons Attribution License (CC-BY 4.0), which permits any use, distribution and reproduction in any medium, provided the original author(s) and source are credited. 


\section{References}

[1] Z. Bern, J.J.M. Carrasco and H. Johansson, New relations for gauge-theory amplitudes, Phys. Rev. D 78 (2008) 085011 [arXiv:0805.3993] [INSPIRE].

[2] Z. Bern, J.J.M. Carrasco and H. Johansson, Perturbative quantum gravity as a double copy of gauge theory, Phys. Rev. Lett. 105 (2010) 061602 [arXiv:1004.0476] [INSPIRE].

[3] Z. Bern, T. Dennen, Y.-t. Huang and M. Kiermaier, Gravity as the square of gauge theory, Phys. Rev. D 82 (2010) 065003 [arXiv: 1004.0693] [INSPIRE].

[4] Z. Bern, L.J. Dixon, D.C. Dunbar, M. Perelstein and J.S. Rozowsky, On the relationship between Yang-Mills theory and gravity and its implication for ultraviolet divergences, Nucl. Phys. B 530 (1998) 401 [hep-th/9802162] [INSPIRE].

[5] M.B. Green, J.H. Schwarz and L. Brink, $N=4$ Yang-Mills and $N=8$ supergravity as limits of string theories, Nucl. Phys. B 198 (1982) 474 [INSPIRE].

[6] Z. Bern, J.S. Rozowsky and B. Yan, Two loop four gluon amplitudes in $N=4$ superYang-Mills, Phys. Lett. B 401 (1997) 273 [hep-ph/9702424] [InSPIRE].

[7] J.J.M. Carrasco and H. Johansson, Five-point amplitudes in $N=4$ Super-Yang-Mills theory and $N=8$ supergravity, Phys. Rev. D 85 (2012) 025006 [arXiv:1106.4711] [INSPIRE].

[8] J.J.M. Carrasco, M. Chiodaroli, M. Günaydin and R. Roiban, One-loop four-point amplitudes in pure and matter-coupled $N \leq 4$ supergravity, JHEP 03 (2013) 056 [arXiv:1212.1146] [INSPIRE].

[9] C.R. Mafra and O. Schlotterer, The structure of n-point one-loop open superstring amplitudes, JHEP 08 (2014) 099 [arXiv: 1203.6215] [INSPIRE].

[10] R.H. Boels, R.S. Isermann, R. Monteiro and D. O'Connell, Colour-kinematics duality for one-loop rational amplitudes, JHEP 04 (2013) 107 [arXiv:1301.4165] [INSPIRE].

[11] N.E.J. Bjerrum-Bohr, T. Dennen, R. Monteiro and D. O'Connell, Integrand oxidation and one-loop colour-dual numerators in $N=4$ gauge theory, JHEP 07 (2013) 092 [arXiv: 1303.2913] [INSPIRE].

[12] Z. Bern et al., Color-kinematics duality for pure Yang-Mills and gravity at one and two loops, Phys. Rev. D 92 (2015) 045041 [arXiv:1303.6605] [INSPIRE].

[13] Z. Bern, S. Davies and T. Dennen, The ultraviolet structure of half-maximal supergravity with matter multiplets at two and three loops, Phys. Rev. D 88 (2013) 065007 [arXiv:1305.4876] [INSPIRE].

[14] J. Nohle, Color-kinematics duality in one-loop four-gluon amplitudes with matter, Phys. Rev. D 90 (2014) 025020 [arXiv: 1309.7416] [INSPIRE].

[15] Z. Bern et al., Ultraviolet properties of $N=4$ supergravity at four loops, Phys. Rev. Lett. 111 (2013) 231302 [arXiv:1309.2498] [INSPIRE].

[16] S.G. Naculich, H. Nastase and H.J. Schnitzer, All-loop infrared-divergent behavior of most-subleading-color gauge-theory amplitudes, JHEP 04 (2013) 114 [arXiv:1301.2234] [INSPIRE].

[17] Y.-J. Du, B. Feng and C.-H. Fu, Dual-color decompositions at one-loop level in Yang-Mills theory, JHEP 06 (2014) 157 [arXiv: 1402.6805] [INSPIRE]. 
[18] C.R. Mafra and O. Schlotterer, Towards one-loop SYM amplitudes from the pure spinor BRST cohomology, Fortsch. Phys. 63 (2015) 105 [arXiv: 1410.0668] [InSPIRE].

[19] Z. Bern, S. Davies and T. Dennen, Enhanced ultraviolet cancellations in $\mathcal{N}=5$ supergravity at four loops, Phys. Rev. D 90 (2014) 105011 [arXiv: 1409.3089] [INSPIRE].

[20] C.R. Mafra and O. Schlotterer, Two-loop five-point amplitudes of super Yang-Mills and supergravity in pure spinor superspace, JHEP 10 (2015) 124 [arXiv: 1505. 02746] [INSPIRE].

[21] S. He, R. Monteiro and O. Schlotterer, String-inspired BCJ numerators for one-loop MHV amplitudes, JHEP 01 (2016) 171 [arXiv:1507.06288] [INSPIRE].

[22] Z. Bern, S. Davies and J. Nohle, Double-copy constructions and unitarity cuts, Phys. Rev. D 93 (2016) 105015 [arXiv: 1510.03448] [INSPIRE].

[23] G. Mogull and D. O'Connell, Overcoming obstacles to colour-kinematics duality at two loops, JHEP 12 (2015) 135 [arXiv: 1511.06652] [INSPIRE].

[24] M. Chiodaroli, M. Günaydin, H. Johansson and R. Roiban, Spontaneously broken Yang-Mills-Einstein supergravities as double copies, JHEP 06 (2017) 064 [arXiv: 1511.01740] [INSPIRE].

[25] Z. Bern et al., Five-loop four-point integrand of $N=8$ supergravity as a generalized double copy, Phys. Rev. D 96 (2017) 126012 [arXiv:1708.06807] [INSPIRE].

[26] H. Johansson and A. Ochirov, Color-kinematics duality for QCD amplitudes, JHEP 01 (2016) 170 [arXiv: 1507.00332] [INSPIRE].

[27] S. Oxburgh and C.D. White, BCJ duality and the double copy in the soft limit, JHEP 02 (2013) 127 [arXiv:1210.1110] [INSPIRE].

[28] C.D. White, Factorization properties of soft graviton amplitudes, JHEP 05 (2011) 060 [arXiv:1103.2981] [INSPIRE].

[29] S. Melville, S.G. Naculich, H.J. Schnitzer and C.D. White, Wilson line approach to gravity in the high energy limit, Phys. Rev. D 89 (2014) 025009 [arXiv:1306.6019] [INSPIRE].

[30] A. Luna, S. Melville, S.G. Naculich and C.D. White, Next-to-soft corrections to high energy scattering in QCD and gravity, JHEP 01 (2017) 052 [arXiv:1611.02172] [INSPIRE].

[31] R. Saotome and R. Akhoury, Relationship between gravity and gauge scattering in the high energy limit, JHEP 01 (2013) 123 [arXiv:1210.8111] [INSPIRE].

[32] A. Sabio Vera, E. Serna Campillo and M.A. Vazquez-Mozo, Color-kinematics duality and the Regge limit of inelastic amplitudes, JHEP 04 (2013) 086 [arXiv:1212.5103] [INSPIRE].

[33] H. Johansson, A. Sabio Vera, E. Serna Campillo and M.A. Vázquez-Mozo, Color-kinematics duality in multi-Regge kinematics and dimensional reduction, JHEP 10 (2013) 215 [arXiv:1307.3106] [INSPIRE].

[34] H. Johansson, A. Sabio Vera, E. Serna Campillo and M.A. Vazquez-Mozo, Color-kinematics duality and dimensional reduction for graviton emission in Regge limit, arXiv:1310.1680 [INSPIRE].

[35] T. Bargheer, S. He and T. McLoughlin, New relations for three-dimensional supersymmetric scattering amplitudes, Phys. Rev. Lett. 108 (2012) 231601 [arXiv:1203.0562] [INSPIRE].

[36] Y.-t. Huang and H. Johansson, Equivalent D $=3$ supergravity amplitudes from double copies of three-algebra and two-algebra gauge theories, Phys. Rev. Lett. 110 (2013) 171601 [arXiv: 1210.2255$]$ [INSPIRE]. 
[37] G. Chen and Y.-J. Du, Amplitude relations in non-linear $\sigma$-model, JHEP 01 (2014) 061 [arXiv: 1311.1133] [INSPIRE].

[38] M. Chiodaroli, Q. Jin and R. Roiban, Color/kinematics duality for general abelian orbifolds of $N=4$ super Yang-Mills theory, JHEP 01 (2014) 152 [arXiv:1311.3600] [INSPIRE].

[39] H. Johansson and A. Ochirov, Pure gravities via color-kinematics duality for fundamental matter, JHEP 11 (2015) 046 [arXiv:1407.4772] [INSPIRE].

[40] H. Johansson and J. Nohle, Conformal gravity from gauge theory, arXiv:1707.02965 [INSPIRE].

[41] M. Chiodaroli, M. Günaydin, H. Johansson and R. Roiban, Gauged supergravities and spontaneous supersymmetry breaking from the double copy construction, Phys. Rev. Lett. 120 (2018) 171601 [arXiv:1710.08796] [INSPIRE].

[42] G. Chen, H. Johansson, F. Teng and T. Wang, On the kinematic algebra for BCJ numerators beyond the MHV sector, JHEP 11 (2019) 055 [arXiv:1906.10683] [INSPIRE].

[43] C. Cheung and G.N. Remmen, Entanglement and the double copy, JHEP 05 (2020) 100 [arXiv: 2002.10470] [INSPIRE].

[44] R. Monteiro, D. O'Connell and C.D. White, Black holes and the double copy, JHEP 12 (2014) 056 [arXiv: 1410.0239] [InSPIRE].

[45] A. Luna, R. Monteiro, D. O'Connell and C.D. White, The classical double copy for Taub-NUT spacetime, Phys. Lett. B 750 (2015) 272 [arXiv:1507.01869] [INSPIRE].

[46] A. Luna et al., The double copy: Bremsstrahlung and accelerating black holes, JHEP 06 (2016) 023 [arXiv: 1603.05737] [INSPIRE].

[47] W.D. Goldberger and A.K. Ridgway, Radiation and the classical double copy for color charges, Phys. Rev. D 95 (2017) 125010 [arXiv:1611.03493] [INSPIRE].

[48] A. Anastasiou et al., Yang-Mills origin of gravitational symmetries, Phys. Rev. Lett. 113 (2014) 231606 [arXiv: 1408.4434] [INSPIRE].

[49] L. Borsten and M.J. Duff, Gravity as the square of Yang-Mills?, Phys. Scripta 90 (2015) 108012 [arXiv: 1602.08267] [INSPIRE].

[50] A. Anastasiou et al., Twin supergravities from Yang-Mills theory squared, Phys. Rev. D 96 (2017) 026013 [arXiv: 1610.07192] [INSPIRE].

[51] A. Anastasiou et al., Are all supergravity theories Yang-Mills squared?, Nucl. Phys. B 934 (2018) 606 [arXiv:1707.03234] [InSPIRE].

[52] G.L. Cardoso, S. Nagy and S. Nampuri, A double copy for $\mathcal{N}=2$ supergravity: a linearised tale told on-shell, JHEP 10 (2016) 127 [arXiv:1609.05022] [INSPIRE].

[53] L. Borsten, $D=6, \mathcal{N}=(2,0)$ and $\mathcal{N}=(4,0)$ theories, Phys. Rev. D 97 (2018) 066014 [arXiv: 1708.02573] [INSPIRE].

[54] A. Anastasiou et al., The Mile High Magic Pyramid, Contemp. Math. 721 (2019) 1 [arXiv: 1711.08476] [INSPIRE].

[55] A. Anastasiou et al., Gravity as gauge theory squared: a ghost story, Phys. Rev. Lett. 121 (2018) 211601 [arXiv: 1807.02486] [inSPIRE].

[56] G. Lopes Cardoso, G. Inverso, S. Nagy and S. Nampuri, Comments on the double copy construction for gravitational theories, PoS (CORFU2017) 177 [arXiv: 1803. 07670] [INSPIRE]. 
[57] W.D. Goldberger, S.G. Prabhu and J.O. Thompson, Classical gluon and graviton radiation from the bi-adjoint scalar double copy, Phys. Rev. D 96 (2017) 065009 [arXiv:1705.09263] [INSPIRE].

[58] W.D. Goldberger and A.K. Ridgway, Bound states and the classical double copy, Phys. Rev. D 97 (2018) 085019 [arXiv:1711.09493] [INSPIRE].

[59] W.D. Goldberger, J. Li and S.G. Prabhu, Spinning particles, axion radiation and the classical double copy, Phys. Rev. D 97 (2018) 105018 [arXiv:1712.09250] [InSPIRE].

[60] A. Luna et al., Perturbative spacetimes from Yang-Mills theory, JHEP 04 (2017) 069 [arXiv: 1611.07508] [INSPIRE].

[61] A. Luna, I. Nicholson, D. O'Connell and C.D. White, Inelastic black hole scattering from charged scalar amplitudes, JHEP 03 (2018) 044 [arXiv:1711.03901] [INSPIRE].

[62] C.-H. Shen, Gravitational radiation from color-kinematics duality, JHEP 11 (2018) 162 [arXiv: 1806.07388] [INSPIRE].

[63] M. Levi, Effective field theories of post-newtonian gravity: a comprehensive review, Rept. Prog. Phys. 83 (2020) 075901 [arXiv: 1807.01699] [INSPIRE].

[64] J. Plefka, J. Steinhoff and W. Wormsbecher, Effective action of dilaton gravity as the classical double copy of Yang-Mills theory, Phys. Rev. D 99 (2019) 024021 [arXiv: 1807.09859] [INSPIRE].

[65] C. Cheung, I.Z. Rothstein and M.P. Solon, From scattering amplitudes to classical potentials in the post-Minkowskian expansion, Phys. Rev. Lett. 121 (2018) 251101 [arXiv: 1808. 02489] [INSPIRE].

[66] M. Carrillo González, R. Penco and M. Trodden, Radiation of scalar modes and the classical double copy, JHEP 11 (2018) 065 [arXiv: 1809.04611] [INSPIRE].

[67] R. Monteiro, I. Nicholson and D. O'Connell, Spinor-helicity and the algebraic classification of higher-dimensional spacetimes, Class. Quant. Grav. 36 (2019) 065006 [arXiv: 1809.03906] [INSPIRE].

[68] J. Plefka, C. Shi, J. Steinhoff and T. Wang, Breakdown of the classical double copy for the effective action of dilaton-gravity at NNLO, Phys. Rev. D 100 (2019) 086006 [arXiv:1906.05875] [INSPIRE].

[69] B. Maybee, D. O'Connell and J. Vines, Observables and amplitudes for spinning particles and black holes, JHEP 12 (2019) 156 [arXiv:1906.09260] [INSPIRE].

[70] H. Johansson and A. Ochirov, Double copy for massive quantum particles with spin, JHEP 09 (2019) 040 [arXiv: 1906.12292] [INSPIRE].

[71] A.P.V. and A. Manu, Classical double copy from color kinematics duality: a proof in the soft limit, Phys. Rev. D 101 (2020) 046014 [arXiv:1907.10021] [INSPIRE].

[72] M. Carrillo González, R. Penco and M. Trodden, Shift symmetries, soft limits and the double copy beyond leading order, arXiv:1908.07531 [INSPIRE].

[73] Y.F. Bautista and A. Guevara, On the double copy for spinning matter, arXiv:1908.11349 [INSPIRE].

[74] N. Moynihan, Kerr-Newman from minimal coupling, JHEP 01 (2020) 014 [arXiv: 1909.05217] [INSPIRE]. 
[75] I. Bah, R. Dempsey and P. Weck, Kerr-Schild double copy and complex worldlines, JHEP 20 (2020) 180 [arXiv: 1910.04197] [INSPIRE].

[76] M. Carrillo González et al., The classical double copy in three spacetime dimensions, JHEP 07 (2019) 167 [arXiv: 1904.11001] [INSPIRE].

[77] W.D. Goldberger and J. Li, Strings, extended objects and the classical double copy, JHEP 02 (2020) 092 [arXiv: 1912.01650] [INSPIRE].

[78] K. Kim, K. Lee, R. Monteiro, I. Nicholson and D. Peinador Veiga, The classical double copy of a point charge, JHEP 02 (2020) 046 [arXiv: 1912.02177] [INSPIRE].

[79] A. Banerjee, E. Colgáin, J.A. Rosabal and H. Yavartanoo, Ehlers as EM duality in the double copy, arXiv:1912.02597 [INSPIRE].

[80] N. Moynihan and J. Murugan, On-shell electric-magnetic duality and the dual graviton, arXiv: 2002.11085 [INSPIRE].

[81] A. Luna, R. Monteiro, I. Nicholson and D. O'Connell, Type D spacetimes and the Weyl double copy, Class. Quant. Grav. 36 (2019) 065003 [arXiv:1810.08183] [INSPIRE].

[82] C.D. White, Exact solutions for the biadjoint scalar field, Phys. Lett. B 763 (2016) 365 [arXiv: 1606.04724] [INSPIRE].

[83] P.-J. De Smet and C.D. White, Extended solutions for the biadjoint scalar field, Phys. Lett. $B \mathbf{7 7 5}$ (2017) 163 [arXiv:1708.01103] [INSPIRE].

[84] N. Bahjat-Abbas, R. Stark-Muchão and C.D. White, Biadjoint wires, Phys. Lett. B 788 (2019) 274 [arXiv: 1810.08118] [InSPIRE].

[85] N. Bahjat-Abbas, R. Stark-Muchão and C.D. White, Monopoles, shockwaves and the classical double copy, JHEP 04 (2020) 102 [arXiv: 2001.09918] [INSPIRE].

[86] D.S. Berman, E. Chacón, A. Luna and C.D. White, The self-dual classical double copy and the Eguchi-Hanson instanton, JHEP 01 (2019) 107 [arXiv: 1809.04063] [INSPIRE].

[87] A.H. Taub, Empty space-times admitting a three parameter group of motions, Ann. Math. 53 (1951) 472.

[88] E. Newman, L. Tamburino and T. Unti, Empty space generalization of the Schwarzschild metric, J. Math. Phys. 4 (1963) 915 [INSPIRE].

[89] G. Bossard, H. Nicolai and K.S. Stelle, Gravitational multi-NUT solitons, Komar masses and charges, Gen. Rel. Grav. 41 (2009) 1367 [arXiv:0809.5218] [INSPIRE].

[90] H. Stephani et al., Exact solutions of Einstein's field equations, Cambridge University Press, Cambridge U.K. (2009).

[91] M. Carrillo-González, R. Penco and M. Trodden, The classical double copy in maximally symmetric spacetimes, JHEP 04 (2018) 028 [arXiv:1711.01296] [INSPIRE].

[92] Z.W. Chong, G.W. Gibbons, H. Lü and C.N. Pope, Separability and Killing tensors in Kerr-Taub-NUT-de Sitter metrics in higher dimensions, Phys. Lett. B 609 (2005) 124 [hep-th/0405061] [INSPIRE].

[93] T.T. Wu and C.N. Yang, Concept of nonintegrable phase factors and global formulation of gauge fields, Phys. Rev. D 12 (1975) 3845 [inSPIRE].

[94] E.J. Weinberg, Classical solutions in quantum field theory, Cambridge Monographs on Mathematical Physics. Cambridge University Press, Cambridge U.K. (2012). 
[95] H.-M. Chan and S.T. Tsou, Some elementary gauge theory concepts, World Sci. Lect. Notes Phys. 47 (1993) 1 [INSPIRE].

[96] P. Goddard, J. Nuyts and D.I. Olive, Gauge theories and magnetic charge, Nucl. Phys. B 125 (1977) 1.

[97] H. Georgi, Lie algebras in particle physics, Front. Phys. 54 (1999) 1.

[98] R.A. Brandt and F. Neri, Stability analysis for singular nonabelian magnetic monopoles, Nucl. Phys. B 161 (1979) 253 [INSPIRE].

[99] L.M. Woodward, The classification of principal pun-bundles over a 4-complex, J. London Math. Soc. s2-25 (1982) 513,

[100] T. Ortín, Gravity and strings, Cambridge University Press, Cambridge U.K. (2004).

[101] R. Alawadhi, D.S. Berman, B. Spence and D. Peinador Veiga, S-duality and the double copy, JHEP 03 (2020) 059 [arXiv: 1911.06797] [INSPIRE].

[102] Y.-T. Huang, U. Kol and D. O'Connell, The double copy of electric-magnetic duality, arXiv: 1911.06318 [INSPIRE].

[103] C. W. Misner, The flatter regions of Newman, Unti, and Tamburino's generalized schwarzschild space, J. Math. Phys. 4 (1963) 924.

[104] C. Hurst, Charge quantization and nonintegrable Lie algebras, Ann. Phys. 50 (1968) 51.

[105] J. Dowker, The NUT solution as a gravitational dyon, Gen. Rel. Grav. 5 (1974) 603.

[106] L.D. Landau and E.M. Lifschits, The classical theory of fields, Course of Theoretical Physics volume 2, Pergamon Press, Oxford U.K. (1975).

[107] P.A.M. Dirac, Quantised singularities in the electromagnetic field,, Proc. Roy. Soc. Lond. A A 133 (1931) 60 [INSPIRE].

[108] J.S. Dowker and J.A. Roche, The gravitational analogues of magnetic monopoles, Proc. Phys. Soc. 92 (1967) 1 [INSPIRE].

[109] U. Kol and M. Porrati, Gravitational Wu-Yang monopoles, Phys. Rev. D 101 (2020) 126009 [arXiv: 2003.09054] [INSPIRE].

[110] H. Godazgar, M. Godazgar and C.N. Pope, New dual gravitational charges, Phys. Rev. D 99 (2019) 024013 [arXiv: 1812.01641] [INSPIRE].

[111] U. Kol and M. Porrati, Properties of dual supertranslation charges in asymptotically flat spacetimes, Phys. Rev. D 100 (2019) 046019 [arXiv: 1907.00990] [InSPIRE].

[112] S. Mandelstam, Quantization of the gravitational field, Annals Phys. 19 (1962) 25 [INSPIRE].

[113] G. Modanese, Wilson loops in four-dimensional quantum gravity, Phys. Rev. D 49 (1994) 6534 [hep-th/9307148] [INSPIRE].

[114] H.W. Hamber and R.M. Williams, Gravitational Wilson loop in discrete quantum gravity, Phys. Rev. D 81 (2010) 084048 [arXiv:0907.2652] [INSPIRE].

[115] A. Brandhuber et al., Four-point amplitudes in $N=8$ supergravity and Wilson loops, Nucl. Phys. B 807 (2009) 290 [arXiv:0805.2763] [InSPIRE].

[116] W. Donnelly and S.B. Giddings, Diffeomorphism-invariant observables and their nonlocal algebra, Phys. Rev. D 93 (2016) 024030 [Erratum ibid. 94 (2016) 029903] [arXiv: 1507.07921] [INSPIRE]. 
[117] S.G. Naculich and H.J. Schnitzer, Eikonal methods applied to gravitational scattering amplitudes, JHEP 05 (2011) 087 [arXiv:1101.1524] [INSPIRE].

[118] D.J. Miller and C.D. White, The gravitational cusp anomalous dimension from AdS space, Phys. Rev. D 85 (2012) 104034 [arXiv:1201.2358] [INSPIRE].

[119] M.B. Green, J.H. Schwarz and E. Witten, Superstring theory. Volume 1: introduction, Cambridge Monographs on Mathematical Physics, Cambridge University Press, Cambridge U.K. (1988).

[120] S. Weinberg, Infrared photons and gravitons, Phys. Rev. 140 (1965) B516 [INSPIRE].

[121] R. Akhoury, R. Saotome and G. Sterman, Collinear and soft divergences in perturbative quantum gravity, Phys. Rev. D 84 (2011) 104040 [arXiv:1109.0270] [InSPIRE].

[122] M. Beneke and G. Kirilin, Soft-collinear gravity, JHEP 09 (2012) 066 [arXiv:1207.4926] [INSPIRE].

[123] E. Gardi and L. Magnea, Infrared singularities in QCD amplitudes, Frascati Phys. Ser. 50 (2010) 137 [arXiv:0908.3273] [InSPIRE].

[124] C.D. White, An introduction to webs, J. Phys. G 43 (2016) 033002 [arXiv:1507.02167] [INSPIRE].

[125] Z. Bern and A.K. Grant, Perturbative gravity from QCD amplitudes, Phys. Lett. B 457 (1999) 23 [hep-th/9904026] [INSPIRE].

[126] I.A. Korchemskaya and G.P. Korchemsky, High-energy scattering in QCD and cross singularities of Wilson loops, Nucl. Phys. B 437 (1995) 127 [hep-ph/9409446] [INSPIRE].

[127] I.A. Korchemskaya and G.P. Korchemsky, Evolution equation for gluon Regge trajectory, Phys. Lett. B 387 (1996) 346 [hep-ph/9607229] [INSPIRE]. 